\title{
A Multi-criteria Decision Support System for Ph.D. Supervisor Selection: A Hybrid Approach
}

\author{
Mir Anamul Hasan and Daniel G. Schwartz \\ Department of Computer Science, Florida State University, Tallahassee, FL, USA

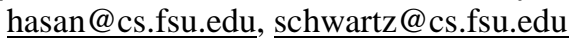

\begin{abstract}
Selection of a suitable Ph.D. supervisor is a very important step in a student's career. This paper presents a multi-criteria decision support system to assist students in making this choice. The system employs a hybrid method that first utilizes a fuzzy analytic hierarchy process to extract the relative importance of the identified criteria and sub-criteria to consider when selecting a supervisor. Then, it applies an information retrieval-based similarity algorithm (TF/IDF or Okapi BM25) to retrieve relevant candidate supervisor profiles based on the student's research interest. The selected profiles are then re-ranked based on other relevant factors chosen by the user, such as publication record, research grant record, and collaboration record. The ranking method evaluates the potential supervisors objectively based on various metrics that are defined in terms of detailed domain-specific knowledge, making part of the decision making automatic. In contrast with other existing works, this system does not require the professor's involvement and no subjective measures are employed.
\end{abstract}

\section{Introduction}

Selection of a Ph.D. supervisor is an important step that a student must take at an early stage in their career. Guidance of the supervisor is a major determiner of quality in a doctoral dissertation [1] and thereby plays a very important role in the student's future success. When deciding whether a particular professor is an appropriate person to serve as supervisor, the student should judge the candidate based on a set of criteria that are important in the supervisor selection process. But identifying the important criteria might be challenging for prospective students due to inexperience. Manderson [2] suggests that students should assess their own needs and the capacities and limitations of potential supervisors, when selecting such a supervisor. Phillips et al. [3] suggests to look for positive answers to at least some of the following questions: "Have they published research papers recently? Do they hold research grants or contracts? Are they invited to speak at conferences in home and abroad?". Students might also be interested in knowing about the quality of journals and conferences where the professors normally publish, their collaborators, their current and previous students' records, etc. Accordingly, students might well take advantage of a decision support system that identifies the important criteria and guides them in evaluating professors with respect to those criteria.

There has been significant research in areas such as research paper recommender systems, collaborator recommendations, expert search, people search, academic search, etc. These address parts of the Ph.D. supervisor selection problem, but research on supervisor selection covering different types of domain-specific knowledge is scant. Several existing works apply methods available in multi-criteria decision analysis, such as the Analytical Hierarchy Process (AHP) [4], the Analytical Network Process (ANP) [1], and COmplex PRoportional ASsessment of alternatives to Grey relations (COPRAS-G) [5] to solve the supervisor selection problem by structuring it as a multiple criteria decision-making problem. All these works assume that students are mature enough and know enough about the potential supervisors in order to perform objective pairwise comparisons of the candidate supervisors on each identified criterion, but this might not be the case all of the time. For example, many of the criteria considered in these works are subjective in nature, such as commitment and involvement [4], relationship with other faculty members [1], or behaving like a "boss" [5]. This makes it impossible to perform pairwise comparisons if the student has not previously interacted with a particular potential supervisor. Some existing works utilize a combination of both subjective and objective measures of different criteria but also have missed important aspects, such as the professor's collaboration network, and do not utilize important details about other potentially relevant criteria, such as citations of papers, recent publications, and research grant details (grant amount, duration, role, etc.) [6] [7]. 
This paper presents a student-centric multi-criteria decision support system for Ph.D. supervisor selection. A set of important criteria to consider is identified and various metrics are defined to evaluate professors objectively with respect to those criteria. The decision support system first retrieves supervisor profiles based on the match between the student's and professors' research areas/interests and then re-ranks candidate supervisor profiles based on several other criteria of interest, selected by the user. The system implements two retrieval algorithms, TF/IDF and Okapi BM25, and lets the user choose the retrieval model to apply when recommending potential supervisors. Students can utilize the system to find a list of potential supervisors based on their research interests and other criteria/subcriteria of interest, concerning a professor's publications, research grants, and collaborators. Then to learn about their personality and availability before making the final decision, the student can contact the professors, inquire with their current and previous students, and meet and take courses with them if that's a possibility.

The paper is structured as follows. Section 2 discusses the related works, section 3 details the underlying method, and section 4 presents details on the developed prototype decision support system. After a discussion of the evaluation methods and results in section 5 , section 6 concludes the paper.

\section{Related Works}

Works closely related to Ph.D. supervisor Recommendation: One of the major concerns when selecting a Ph.D. supervisor is to find professors who work in the area of student's research interests. There has been significant work in the field of research paper recommender systems, where relevant papers are recommended based on some form of inputs. Some of the existing works in research paper recommender systems have utilized user-provided keywords, text snippets, parts of a research paper of interest (such as, title, abstract, bibliography, etc.), or the entire paper, as input to generate recommendations [8]. Some have employed papers that the users had authored [9], tagged [10], browsed [11], or downloaded [12].

Significant research has been done in the field of collaborator recommendation for scholars. Existing works utilize the user's research interest [13], publications and co-author network [13] [14] [15], academic homepages [14], temporal evolution of research interest, comparative seniority status [16], and so on, to find potential collaborators. Target users of these studies are normally professors in academia or researchers in enterprises, and not $\mathrm{Ph}$. D. students who are looking for supervisors with whom to work.
Moreover, most of these studies do not evaluate how influential a professor's existing collaboration network might be, which also could be of interest to the students.

There have also been works in the field of expert/ people search, and academic search to find experts in a particular topic [17] [18]. These are not student centric and cover only parts of the supervisor selection problem.

Works in Ph.D. Supervisor Recommendation: Several existing works have structured the selection problem of Ph.D. supervisor as a multiple criteria decision making (MCDM) problem and applied different methods used in multi-criteria decision analysis to solve it. Ray [4] demonstrated the use of AHP in the selection of doctoral dissertation supervisors. Momeni et al. [1] used ANP in Ph.D. supervisor selection. ANP allows interdependencies among the decision attributes, whereas AHP assumes selection criteria are independent. Datta et al. [5] used another method employed in multi-criteria decision analysis, called COPRAS-G, to select a suitable supervisor. All these works have followed a similar research methodology, where doctoral students were first interviewed to collect a list of factors to consider before selecting a supervisor, and then they were interviewed again to ascertain the relative weights of those factors through pairwise comparison. Then pairwise comparison of the alternatives, i.e. the potential supervisors, is performed with respect to each of the criteria, and finally the alternatives are ranked using a synthesis process.

Zhang et al. [6] presented a Research Analytics Framework for Education (RAF-E), this being a student centric method for finding and recommending supervisors for new postgraduate students, considering different metrics from 3 dimensions: relevance, connectivity, and quality. Zhang et al. [7] proposed a personality-matching aided approach for supervisor recommendation based on their previous work [6], which integrates objective measurements (relevance, connectivity, and quality) and subjective personality matching, to get a list of supervisors to recommend.

Alarfaj et al. [19] proposed an information-retrieval based supervisor recommendation method, which returns ranked results based on frequency of candidate supervisor name and proximity of user query and supervisor name in pages returned for a user query by an underlying search engine.

The aforementioned works [1] [4] [5] have used purely subjective measures of different criteria, and [6] [7] [19] have employed objective measures but have missed important details and did not consider some of the important criteria as discussed in the foregoing Introduction. Moreover, these works either create supervisor profiles by interviewing professors or require that professors create their own profiles. 


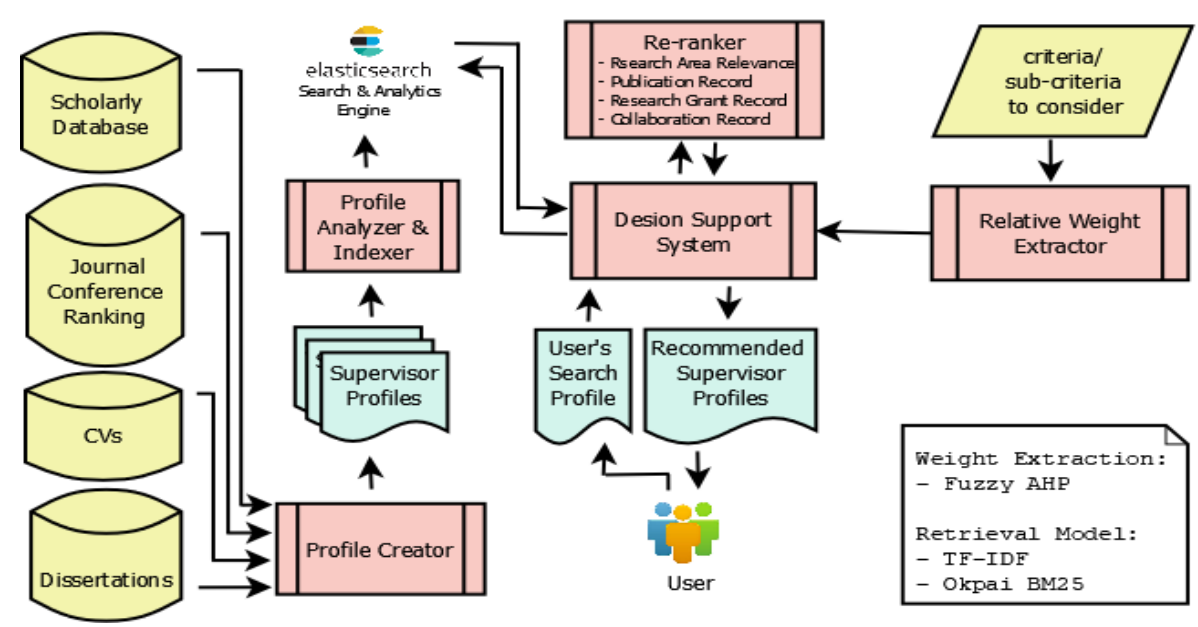

Figure 1. System Architecture of the Proposed Decision Support System

\section{Proposed Method}

The multi-criteria decision support system being proposed here helps students in the selection process of a Ph.D. supervisor by guiding them in identifying and selecting important criteria/sub-criteria to consider and recommending potential supervisors based on that selection. An overview of the proposed method is given in Figure 1.

First, important decision variables, i.e. criteria/subcriteria to consider, are identified when selecting a Ph.D. supervisor. Then relative weights of those decision variables are calculated through pairwise comparison applying fuzzy AHP by conducting a survey among graduate students. Then documents, i.e. supervisor profiles, are created, collecting data from various relevant sources with respect to those identified criteria/sub-criteria, and then those supervisor profiles are analyzed and indexed in Elasticsearch [20], a document-oriented NoSQL database. To get recommendations, users need to complete a search profile, where they can enter their research interests and custom select the decision variables they think are important in the selection process. The decision support system first retrieves relevant supervisor profiles from the indexed documents based on the user's research interests given in the search profile, utilizing an IR based similarity algorithm (TF/IDF or Okapi BM25) and then re-ranks those candidate supervisor profiles based on the selected criteria/sub-criteria of interest in the search profile, and suggests them to the user. We defined various metrics to objectively measure the identified decision variables and employed the extracted weights of the decision variables in different phases of the final rank computation process.

\subsection{Identifying Decision Variables}

We identified important factors to consider when selecting a Ph.D. supervisor on the basis of the intensive review of the available prior research in the relevant fields discussed in section 2, together with an analysis the complexities and challenges encountered during the $\mathrm{Ph}$.D. supervisor selection process, and then developed a hierarchical structure for the Ph.D. supervisor selection problem. We identified four main criteria to consider, namely, research area relevance, publication record, research grant record, and collaboration record. These criteria are then further broken down into various sub-criteria, which are presented in Figure 2. In the hierarchy, the overall objective/goal is placed at level 1 , criteria at level 2, sub-criteria at level 3, and the decision alternatives at level 4. Additional details regarding the identified criteria and sub-criteria are discussed in section 3.3.

\subsection{Determining Weights of Different Criteria and Sub-criteria}

AHP is a widely used tool for solving complex multiple criteria decision-making problem involving subjective judgment. Introduced by Saaty [21], this has previously been used in one of the related works concerning Ph.D. supervisor selection [4]. In AHP, weights are calculated via pairwise comparisons of both the criteria and alternatives on a relative importance scale of 1 to 9 . As the conventional AHP does not include vagueness for subjective judgements, many studies have included different techniques into AHP to accommodate vagueness, such as fuzzy set theory [22] [23], probability theory [24] [25], and numeric interval 


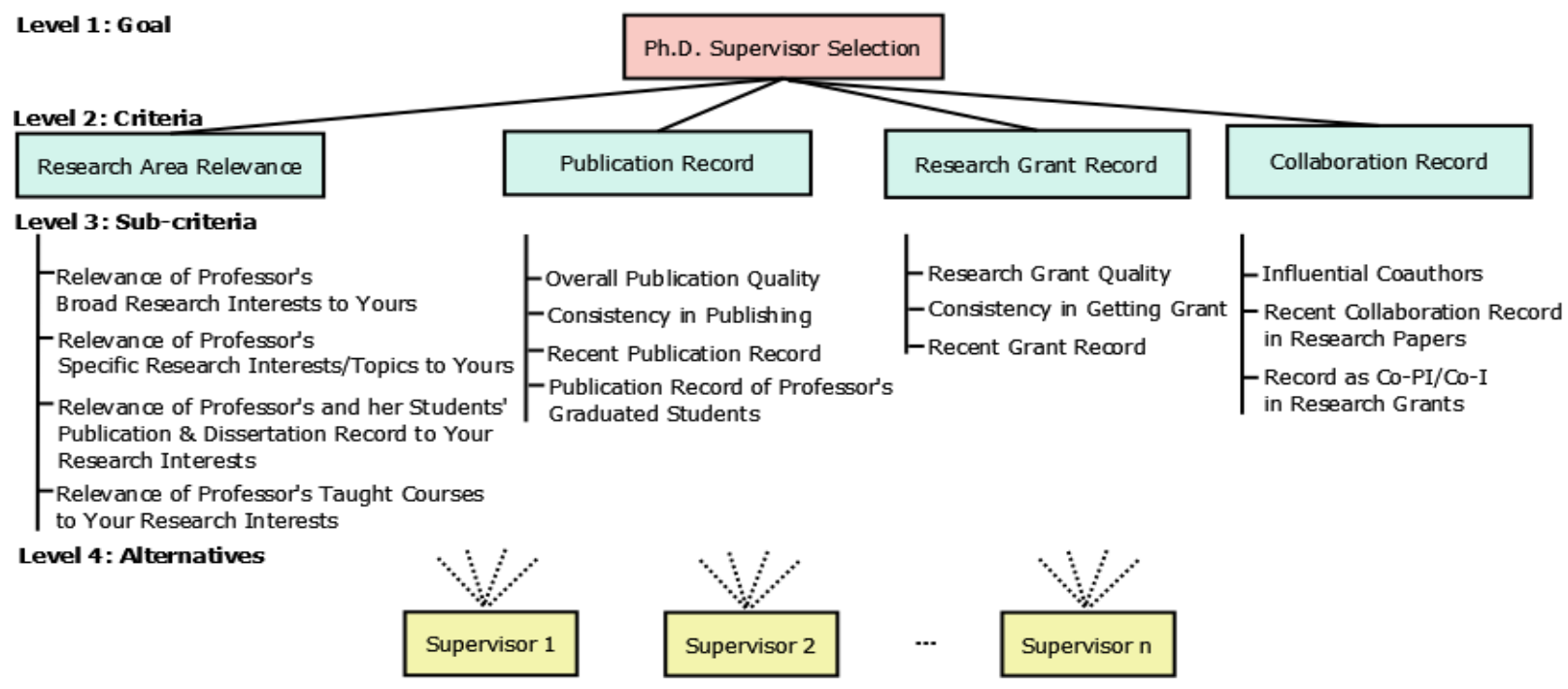

Figure 2. Hierarchical Structure of the Ph.D. Supervisor Selection Problem

estimates [26] [27]. In this study, we used Fuzzy AHP to determine the relative weights of the identified criteria and sub-criteria, since this approach is adequate to explicitly capture the importance assessment for imprecise human judgments [28]. This technique has not previously been used in our problem domain, i.e., selection of a Ph.D. supervisor.

Fuzzy AHP provides a systematic approach to solve multi-criteria decision problem by using the concepts of fuzzy set theory (developed by Zadeh [29]) and hierarchical structure analysis. Many Fuzzy AHP methods have been proposed by various authors. For this study, we used Ayhan's [23] implementation of Buckley's method [22] to determine the relative importance of the identified criteria/sub-criteria. This introduces triangular fuzzy numbers into the conventional AHP in order to enhance the degree of judgment of the decision maker.

A triangular fuzzy number is a special fuzzy set $\tilde{F}$ in a universe of discourse $U$ and can be defined as $\tilde{F}=(1, \mathrm{~m}, \mathrm{u})$, where $\mathrm{l}$ and $\mathrm{u}$ stand for lower and upper value of $\tilde{F}$ and $\mathrm{m}$ is the mid-value of $\tilde{F}$. The symbol ' $\sim$ ' on a letter is used to indicate that the letter represents a fuzzy set. The membership function $\mu_{\tilde{F}}(\mathrm{x})$, which associates a real number in the interval $[0,1]$ with each element $x$ in $\mathrm{X}$, to represent the grade of membership of $\mathrm{x}$ in $\tilde{F}$ is defined as [30]:

$$
\mu_{\tilde{F}}(\mathrm{x})= \begin{cases}\frac{x-l}{m-l}, & l \leq x \leq m \\ \frac{u-x}{u-m}, & m \leq x \leq u \\ 0, & \text { otherwise }\end{cases}
$$

The corresponding linguistic terms and triangular fuzzy number representation of Saaty's 1 to 9 relative importance scale is depicted in Table 1 .
Table 1. Fuzzy Version of Saaty's 1 to 9 Scale

\begin{tabular}{|c|c|c|}
\hline $\begin{array}{l}\text { Satty's } \\
\text { Scale }\end{array}$ & Linguistic Terms & $\begin{array}{l}\text { Triangular } \\
\text { Fuzzy } \\
\text { Scale }\end{array}$ \\
\hline 1 & Equally important & $(1,1,1)$ \\
\hline 2 & $\begin{array}{l}\text { Equally to slightly more } \\
\text { important }\end{array}$ & $(1,2,3)$ \\
\hline 3 & Slightly more important & $(2,3,4)$ \\
\hline 4 & $\begin{array}{l}\text { Slightly to significantly } \\
\text { more important }\end{array}$ & $(3,4,5)$ \\
\hline 5 & Significantly more important & $(4,5,6)$ \\
\hline 6 & $\begin{array}{l}\text { Significantly to very } \\
\text { significantly more important }\end{array}$ & $(5,6,7)$ \\
\hline 7 & $\begin{array}{l}\text { Very significantly more } \\
\text { important }\end{array}$ & $(6,7,8)$ \\
\hline 8 & $\begin{array}{l}\text { Very significantly to } \\
\text { absolutely more important }\end{array}$ & $(7,8,9)$ \\
\hline 9 & Absolutely more important & $(9,9,9)$ \\
\hline
\end{tabular}

We conducted a survey among computer science graduate students to get the preferences of one criteria/sub-criteria over the other through pairwise comparison. First, the relative weights of each criteria (research area, publication record, research grant record, and collaboration record) are determined. The steps of the procedure are as follows.

The pairwise comparison matrix, $\widetilde{P}$ is computed as:

$$
\tilde{P}=\left[\begin{array}{cccc}
\widetilde{s_{11}} & \widetilde{s_{12}} & \cdots & \widetilde{s_{1 n}} \\
\vdots & \widetilde{s_{22}} & \cdots & \widetilde{s_{2 n}} \\
\widetilde{s_{n 1}} & \widetilde{s_{n 2}} & \cdots & \vdots \\
\widetilde{s_{n n}}
\end{array}\right]
$$

where $\widetilde{s_{l j}}$ is the averaged preferences of $i$-th criterion over j-th criterion, defined as $\widetilde{s_{l j}}=\frac{\sum_{k=1}^{K} \widetilde{s_{l j}^{k}}}{K}$, where $\widetilde{s_{l j}^{k}}$ represents the k-th survey taker's preference of the i-th 
criterion over the $\mathrm{j}$-th criterion, and $\mathrm{K}$ is the total number of valid survey responses. After that, the geometric mean of the fuzzy comparison values of each criterion is computed as: $\widetilde{r}_{l}=\left(\prod_{j=1}^{n} \widetilde{S_{l \jmath}}\right)^{1 / n}$. Then, the fuzzy weight of each criterion $\widetilde{w_{l}}$ is computed as $\widetilde{w_{l}}=\widetilde{r}_{l}$ $\otimes\left(\widetilde{r_{1}} \oplus \widetilde{r_{2}} \oplus \ldots \oplus \widetilde{r_{n}}\right)^{-1}$, where $\oplus$ and $\otimes$ are the fuzzy addition and multiplication operators. Then, the fuzzy weights $\left(\widetilde{W_{l}}\right)$ are de-fuzzified to $M_{i}$, where $M_{i}$ is the non-fuzzy weight of each criterion and defined as $\mathrm{M}_{\mathrm{i}}$ $=\frac{l w_{i}+m w_{i}+u w_{i}}{3}$. Finally, the non-fuzzy weight $\mathrm{M}_{\mathrm{i}}$ is normalized to $\mathrm{N}_{\mathrm{i}}$ to get the final weights of each criterion. $\mathrm{N}_{\mathrm{i}}$ is defined as: $\mathrm{N}_{\mathrm{i}}=\frac{M_{i}}{\sum_{i=1}^{n} M_{i}}$. We follow the same procedure to extract the relative importance of each sub-criteria under each of those criteria. Details about the survey are discussed in section 4 .

\subsection{Evaluating the Alternatives}

In conventional AHP and Fuzzy AHP, the relative importance of alternatives is calculated through pairwise comparison of the alternatives with respect to the identified criteria and sub-criteria and then alternatives are ranked based on a synthesis process. In the proposed hybrid method, we define metrics (see the following) for the identified criteria/sub-criteria, which makes it possible to evaluate the alternatives objectively and automatically. This differs from typical AHP and Fuzzy AHP applications where the alternatives are evaluated and compared manually.

The proposed two-phase decision support system provides the user with a search interface as depicted in Figure 3. Here the user can enter text data regarding their research interests, specific research interest, title and abstract of a paper of interest, and select the criteria/sub-criteria of their interest. In the first phase, the proposed method retrieves matching candidate supervisor profiles based on relevance between the student's and supervisors' research areas/interests. Then in the second phase, the candidate supervisor profiles are re-ranked based on the selected criteria/sub-criteria of interest and presented to the user.

3.3.1. Research Area Relevance $\left(\mathbf{C}_{1}\right)$. The research area relevance is evaluated based on the following four sub-criteria: relevance of the professor's broad research interests to those of the Student $\left(C_{11}\right)$, relevance of the professor's specific research interests/topics to those of the student $\left(C_{12}\right)$, relevance of the professor's and their previous students' publication and dissertation record to the student's research interests $\left(C_{13}\right)$, and relevance of the professor's previously taught courses to the student's research interests $\left(C_{14}\right)$.

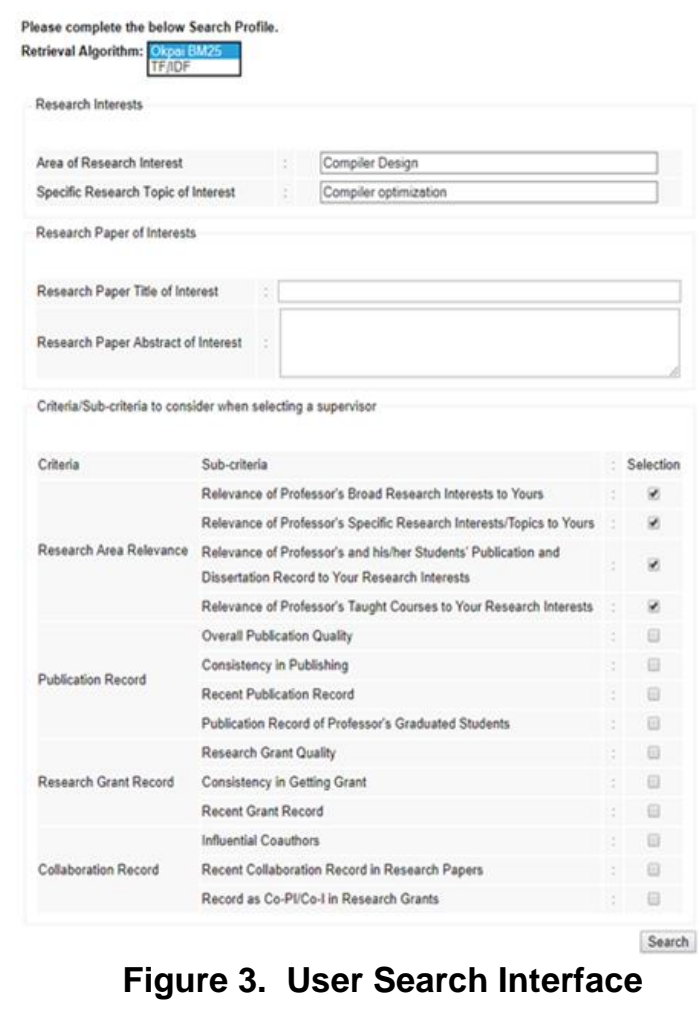

The proposed method first retrieves candidate supervisor profiles based on research area relevance. The retrieval model in the proposed system implements two information-retrieval based similarity algorithms, TF/IDF and Okapi BM25, and lets the user choose between the two approaches.

TF/IDF. The TF/IDF similarity algorithm utilizes the term frequency-inverse document frequency (tf-idf) weighting scheme, which is developed based on the following empirical observations regarding text [31]:

- IDF assumption: Rare terms are not less relevant than frequent terms.

- TF assumption: Multiple occurrences of a term in a document are not less relevant than single occurrences.

- Normalization assumption: Long documents are not preferred to short documents.

So, a term that appears frequently in a document, but rarely appears in other documents in the document collection, is more likely to represent the topic of the document. And normalizing the resulting weight vectors makes sure that longer documents don't have a better chance of retrieval.

In the TF-IDF similarity algorithm, the relevance score of a document $d$ for query $q$ which consists of terms $\mathrm{t}$ is defined as [32]:

$$
\operatorname{score}(d, q)=\sum_{t \text { in } q}\left(t f(t \text { in } d) \operatorname{idf}(t)^{2} \operatorname{norm}(d)\right)
$$


where $\operatorname{tf}(\mathrm{t}$ in $\mathrm{d})$ is the term frequency of term $\mathrm{t}$ in document $\mathrm{d}$ and computed as $\mathrm{t} f(\mathrm{t}$ in $\mathrm{d})=\sqrt{\text { frequncy, }}$ $\operatorname{idf}(t)$ is the inverse document frequency of term $t$ and computed as idf $(t)=1+\log \frac{\text { numDocs }}{\operatorname{docFreq}(t)+1}$, where numDocs is the number of all documents in the collection and docFreq $(t)$ is the number of documents containing term $\mathrm{t}$, and norm( $\mathrm{d})$ is the normalization factor of a matching document $d$, which causes higher weights for short documents, computed as norm $(\mathrm{d})=\frac{1}{\sqrt{\text { numTerms }}}$.

Okapi BM25. Okapi BM25 is a similarity algorithm to score matching documents according to their relevance to a search query and is developed based on the probabilistic retrieval model [33]. In the Okapi BM25 similarity algorithm, the relevance score of a document $d$ for query $q$, which consists of terms $t$ is defined as [32]:

$\operatorname{score}(d, q)$

$=\sum_{t \text { in } q}\left(\operatorname{idf}(\mathrm{t}) \frac{t f(t \text { in } d)(k+1)}{t f(t \text { in } d)+k\left(1-b+b \frac{|D|}{a v g d l}\right)}\right)$

where $t f(t$ in $d)$ is the number of occurrences of term $t$ in document $d,|D|$ is the document length, avgdl is the average document length over all documents in the collection, $\operatorname{idf}(\mathrm{t})$ for term $\mathrm{t}$ is computed as idf $(\mathrm{t})=$ $\log \left(1+\frac{\text { numDocs }- \text { docFreq }(t)+0.5}{\text { docFreq }(t)+0.5}\right)$, where numDocs is the number of documents in the collection and docFreq $(\mathrm{t})$ is the number of documents containing term $\mathrm{t}$, and $\mathrm{k}$ and $\mathrm{b}$ are the tuning parameters. In our experiment, we used BM25 with standard values for $\mathrm{k}(1.2)$ and $\mathrm{b}(0.75)$ [32].

We utilized the Elasticsearch's [20] implementations of the TF/IDF and Okpai BM25 similarity algorithms to retrieve relevant documents. We wrote a multi-field search query following the Elasticsearch query DSL [20], where matches in broader research interests (i.e. $C_{11}$ ) is boosted with $\omega_{11}$, matches in specific research interests (i.e. $C_{12}$ ) is boosted with $\omega_{12}$, matches in publication record (i.e. $C_{13}$ ) is boosted with $\omega_{13}$, and matches in taught courses (i.e. $C_{14}$ ) is boosted with $\omega_{14}$, Here, $\omega_{11}, \omega_{12}, \omega_{13}$ and $\omega_{14}$ are the relative weights of the sub-criteria. The weights of the identified criteria/sub-criteria are calculated following the steps discussed in section 3.2 and actual weights used in our system are given in section 4 .

3.3.2. Publication Record $\left(\mathbf{C}_{2}\right)$. Academic performance of professors is often measured in terms of number of publications and the quality of journals/conferences where they were published [34] [35]. The citation count of a paper can give a rough idea of the paper's popularity [8].
The factors (sub-criteria) that can affect the publication record criterion are as follows.

Overall Publication Quality $\left(C_{21}\right)$. The overall publication quality is defined as:

$$
\mathrm{C}_{21}=\frac{\sum_{i=1}^{N^{p}} c c_{i}^{p} r_{i}^{p}}{\sum_{i=1}^{N^{p}} r_{i}^{p}}
$$

where, $N^{p}=$ total no. of papers, $c c_{i}^{p}=$ no. of citations of the $\mathrm{i}$-th paper, and $r_{i}^{p}=$ rank of the journal or conference in which the i-th paper is published according to the CORE ranking database (www.core.edu.au). The CORE ranking database provides rankings of conferences and journals in the computing disciplines.

Consistency in Publishing $\left(C_{22}\right)$. Another important aspect to consider when evaluating a professor's publication record is to check how consistent they are in publishing throughout their publishing career [3]. The consistency in publishing measure is defined as:

$$
\mathrm{C}_{22}=1-\frac{Y^{\text {wop }}}{Y^{c}-Y^{f p}}
$$

where, $Y^{w o p}=$ no. of years without publication, $Y^{c}=$ current year, and $Y^{f p}=$ year of first published paper.

Recent Publication Record $\left(C_{23}\right)$. The recent publication record of a professor is a good indicator of whether a professor is active in research or not, as well as the direction and quality of their current research [3]. The recent publication record measure is defined as

$$
\mathrm{C}_{23}=n^{p}+\left(\frac{n^{p A}}{n^{p}}+\frac{n^{p A+p B}}{n^{p}}\right)
$$

where, $n^{p}=$ no. of papers published in last five years, $n^{p A}=$ no. of papers published in type $\mathrm{A}$ journals or conferences in the last five years, $n^{p B}=$ no. of papers published in type $\mathrm{B}$ journals or conferences in the last five years. The types/ ranks of journals and conferences are extracted from the CORE ranking database (www.core.edu.au).

Publication Record of Professor's Graduated Students $\left(C_{24}\right)$. The publication record of graduated students of a professor might be of interest to the students, as some might want to be employed in academia/research organizations, where quality and number of publications matter. This sub-criterion is defined as: $C_{24}=\mathrm{M}(\mathrm{G})$, i.e. the median of $G$, where $G$ is a set of numbers representing the number of publications of graduated students. The median is used as we assume the sample data size will be small.

Finally, overall publication quality is evaluated as:

$$
\mathrm{C}_{2}=\omega_{21} C_{21}^{\prime}+\omega_{22} \mathrm{C}_{22}+\omega_{23} C_{23}^{\prime}+\omega_{24} C_{24}^{\prime}
$$

where $\omega_{21}, \omega_{22}, \omega_{23}$ and $\omega_{24}$ are the relative weights of the sub-criteria. A metric above and hereafter with the symbol "' is assumed to be normalized by scaling it into the range $[0,1]$ based on the corresponding values of the candidate supervisor profiles. 
3.3.3. Research Grant Record $\left(\mathbf{C}_{3}\right)$. Typically, a Ph.D. student is supported through graduate assistantship, be it teaching or research, which is generally viewed as a means of enhancing the professional development of the student, in addition to providing financial support [36]. These positions are time demanding. Teaching assistants are normally assigned menial types of duties, such as checking assignments and grading tests and quizzes, with occasional greater opportunities for professional development through teaching a course by assuming full responsibility. But those have little or nothing to do with student's success/progress on their Ph.D. dissertation research. On the other hand, research assistants get the opportunity to be involved in the design and conduct of exciting funded research projects, which helps them develop valuable research skills needed for their graduate study and future career and, in most of the cases, those works become part of their dissertation. A study by Wong et al. [37] found that receipt of a teaching assistantship is less likely to be associated with graduate success than receipt of a research assistantship.

So, the professor's grant record might be of interest to the students, as research assistants are usually supported through the grant money of professor's active research grants, funded by different funding agencies. Moreover, research by Bozeman et al. [38] found that professors with more grants and contracts of each type (government and industry) have a greater propensity for industrial involvement than those who have fewer such contracts. A professor's connection with industry people might also be of interest to some students for future opportunities, like, internships or full-time jobs after graduation.

To evaluate a professor's research grant record, the following three sub-criteria are identified.

Research Grant Quality $\left(C_{31}\right)$. Research grant quality is evaluated in terms of grant duration, grant amount, and the role played by the professor (PI, Co-PI, etc.) and is defined as: $\quad \mathrm{C}_{31}=\frac{\sum_{i=1}^{N g} d_{i}^{g} a_{i}^{g} r_{i}^{g}}{\sum_{i=1}^{N g} a_{i}^{g} r_{i}^{g}}$

where, $N^{g}=$ total no. of grants, $d_{i}^{g}=$ duration of i-th grant, $a_{i}^{g}=$ amount of i-th grant, and $r_{i}^{g}=$ role in the ith grant, which works as a boosting factor. We set $r_{i}^{g}=1$, if the role is Co-PI and $r_{i}^{g}=2$, if the role is PI.

Consistency in Getting Grants $\left(C_{32}\right)$. Consistency in getting grants is also taken into consideration when evaluating the research grant record and is defined as:

$$
\mathrm{C}_{32}=1-\frac{Y^{\text {wog }}}{Y^{c}-Y^{f g}}
$$

where, $Y^{\text {wog }}=$ no. of years without a grant, $Y^{c}=$ current year, and $Y^{f g}=$ year of the first grant received.

Recent Grant Record $\left(C_{33}\right)$. Recent grant record is taken into considerations, as professors who have active grants and contracts, are more likely to be productive [38] and support students as research assistants. This is defined as: $\quad \mathrm{C}_{33}=n_{g}+n_{c}^{g}$ where, $n_{g}=$ no. of research grants in last 5 years, and $n_{c}^{g}=$ no. of current grants.

Finally, the overall research grant record is evaluated as:

$$
\mathrm{C}_{3}=\omega_{31} C_{31}^{\prime}+\omega_{32} \mathrm{C}_{32}+\omega_{33} C_{33}^{\prime}
$$

where $\omega_{31}, \omega_{32}$, and $\omega_{33}$ are the relative weights of the sub-criteria.

3.3.4. Collaboration Record $\left(\mathbf{C}_{4}\right)$. Another aspect to consider when selecting a Ph.D. supervisor is the professor's collaboration record. Collaboration tends to have positive effects on research productivity [39]. Analyzing 592 scientists' publications and collaborative activities, Price et al. [40] found that "The most prolific author is also by far the most collaborating, and three of the four next most prolific are also among the next most frequently collaborating". Working with a professor who has a strong collaboration network might give students the opportunity to be involved in exciting collaborative projects, thereby providing students with the opportunity to interact with and learn from the professor's collaborators, as well as create new connections in academia/industry. Three sub-criteria are identified that affect the collaboration record criterion. Influential Co-authors $\left(C_{41}\right)$. To assess the list of coauthors of a professor, we considered the reputation of the co-authors in terms of citations [41] and number of times they have co-authored [41], and give more importance to recent co-authorship. $\mathrm{C}_{41}$ is defined as:

$$
\mathrm{C}_{41}=\frac{\sum_{i=1}^{N^{c A}} b n_{c A_{i}}^{p} \log \left(c c_{c A_{i}}^{t}\right)}{\sum_{i=1}^{N^{c A}} b n_{c A_{i}}^{p}}
$$

where $N^{c A}=$ total no. of co-authors, $n_{c A_{i}}^{p}=$ no. of coauthored papers with co-author i, $c c_{C A_{i}}^{t}=$ total number of citations of co-author $\mathrm{i}, \mathrm{b}=$ boost factor, which boosts recent involvement ( $b$ will be no. of co-authored paper in last five years).

Recent Collaboration Record in Research Papers $\left(C_{42}\right)$. This is defined as: $C_{42}=$ no. of co-authors in last 5 years. Record as Co-PI/Co-I in Research Grants $\left(C_{43}\right)$. This is defined as: $C_{43}=$ no. of research grants as co-PI/co-I in last 5 years.

Finally, the overall collaboration record is evaluated as: $\quad \mathrm{C}_{4}=\omega_{41} C_{41}^{\prime}+\omega_{42} C_{42}^{\prime}+\omega_{43} C_{43}^{\prime}$

where $\omega_{41}, \omega_{42}$, and $\omega_{43}$ are the relative weights of the sub-criteria.

Once we get the corresponding scores for research area relevance $\left(\mathrm{C}_{1}\right)$, publication record $\left(\mathrm{C}_{2}\right)$, research grant record $\left(\mathrm{C}_{3}\right)$ and collaboration record $\left(\mathrm{C}_{4}\right)$ for the candidate supervisor profiles, the final recommendation score for the potential supervisors is computed as: 


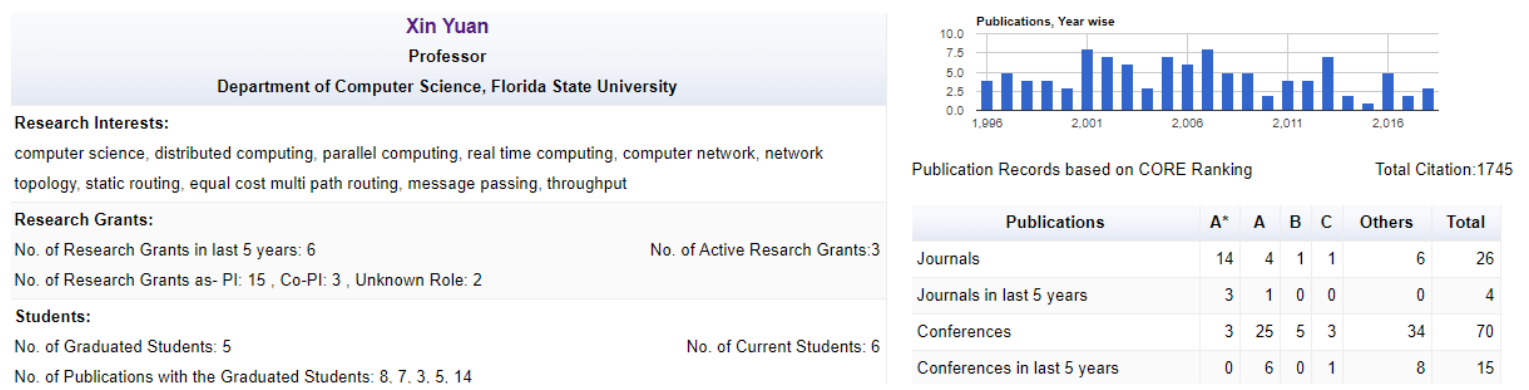

Figure 4. Result Interface of the Proposed Decision Support System

$$
R=\left[\begin{array}{c}
r_{1} \\
r_{2} \\
\vdots \\
r_{n}
\end{array}\right]=\left[\begin{array}{c}
\omega_{1} \\
\omega_{2} \\
\omega_{3} \\
\omega_{4}
\end{array}\right]\left[\begin{array}{cccc}
C_{1}^{1} & C_{2}^{1} & C_{3}^{1} & C_{4}^{1} \\
C_{1}^{2} & C_{2}^{2} & C_{3}^{2} & C_{4}^{2} \\
\vdots & \vdots & \vdots & \vdots \\
C_{1}^{n} & C_{2}^{n} & C_{3}^{n} & C_{4}^{n}
\end{array}\right]
$$

where, $\mathrm{n}$ is the number of candidate supervisor profiles, $r_{i}$ is the final recommendation score of the $i$-th candidate supervisor profile, where $i=1,2, \ldots, n$, and $\omega_{1}, \omega_{2}, \omega_{3}$, and $\omega_{4}$ are relative weights of the four main criteria.

\section{System Implementation}

A prototype decision support system has been developed to evaluate the proposed method. First, a dataset of 54 professors from three different departments in the computing disciplines (Computer Science, Electrical and Computer Engineering, and Scientific Computing) at Florida State University (FSU) is created by crawling and parsing data from four different sources: (i) publications data are collected from Microsoft Academic [42], a scholarly database, (ii) rankings of journals and conferences are extracted from the CORE ranking database, (iii) $\mathrm{CVs}$ of the professors are parsed to extract research grant information, current and previous students information, and lists of courses taught, which are available at a public $\mathrm{CV}$ database (www.fsu.edu/cvdb) and (iv) dissertation information (title, abstract) of the professor's graduated students is collected from DigiNole (https://fsu.digital.flvc.org/), FSU's digital repository. We index the supervisor profiles twice in two different indices in Elasticsearch, once for the TF/IDF based retrieval model and once for the Okapi BM25, as they are analyzed differently. We developed the web-based decision support system using J2EE technologies.

Based on the user input in the user search profile depicted in Figure 3, the decision support system retrieves a list of potential supervisors from the indexed supervisor profiles and displays them in the result interface depicted in Figure 4. The potential supervisors are presented with useful information covering their research area, publication record, research grants, and collaboration record. The user can also check individual supervisor profiles. The supervisor profiles are very rich, providing useful detailed information about each professor with graphs and charts, when relevant, and can thereby help the user make a more informed decision. More details are reported in a demonstration paper [47].

In the system implementation, we used relative weights of identified criteria and sub-criteria when computing the recommendation score. We conducted a survey to extract those weights, where participants (computer science graduate students in the host department as well as other US universities) were first asked to do pairwise comparison of the identified criteria and then do pair-wise comparisons of the subcriteria under each criterion. We collected 28 valid survey responses, where 25 survey respondents were Ph.D. students, 3 were M.S. students, and 20 of the 25 $\mathrm{Ph}$.D. students had already selected their supervisor. The weights were extracted following the steps described in section 3.2. The corresponding values are $\omega_{1}=0.54, \omega_{2}=0.27, \omega_{3}=0.12, \omega_{4}=0.07 ; \omega_{11}=0.57, \omega_{12}$ $=0.25, \omega_{13}=0.13, \omega_{14}=0.05 ; \omega_{21}=0.53, \omega_{22}=0.27, \omega_{23}$ $=0.14, \omega_{24}=0.07 ; \omega_{31}=0.65, \omega_{32}=0.24, \omega_{33}=0.12$ and $\omega_{41}=0.65, \omega_{42}=0.26, \omega_{43}=0.08$.

\section{Evaluation}

To evaluate the quality of the recommendations generated by the proposed method, we asked $20 \mathrm{Ph} . \mathrm{D}$. students in the host department to rate the recommendations on a scale of four: not relevant (0), somewhat relevant (1), relevant (2) and very relevant (3) in three different settings (baseline, custom, all) for each retrieval algorithm (TF/IDF, Okpai BM25). The three settings are defined as follows.

Baseline: In the baseline method, recommendations are made based purely on research area relevance.

Custom: In the custom method, the user can custom select their criteria/sub-criteria of interest, based on which the recommendations will be generated.

All: In the all method, all criteria/sub-criteria will be considered when computing the recommendation score.

We used Average Rate (AR) [6] and Normalized Discounted Cumulative Gain (NDCG) [6] as the 
evaluation metrics. Table 2 reports AR values of the user ratings for both retrieval models in the three different settings. It can be easily observed that for both retrieval models, the Custom setting of criteria/subcriteria performs better, and between the TF/IDF and BM25 algorithms, in most cases, the BM25 based algorithm performs better. Table 3 reports the NDCG values, where also Custom settings of the criteria/subcriteria-based configuration performs better than the Baseline and All settings for both the TF/IDF and BM25 based retrieval models. So, analysis of the results reveals that letting the user custom select the criteria/sub-criteria of interest provides more satisfactory recommendations compared to the Baseline and All criteria/sub-criteria selection settings.

Table 2. Performance Comparison: AR

\begin{tabular}{|c|c|c|c|c|c|c|}
\hline Evaluation & \multicolumn{2}{|c|}{ AR@1 } & \multicolumn{2}{|c|}{ AR@2 } & \multicolumn{2}{|c|}{ AR@3 } \\
\hline $\begin{array}{l}\text { Retrieval } \\
\text { Model } \\
\text { Criteria } \\
\text { Setting } \mathbf{}\end{array}$ & 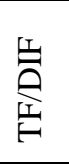 & $\sum_{\infty}^{n}$ & $\underset{⿱ 乛}{\stackrel{I}{\mid}}$ & 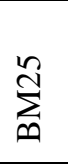 & 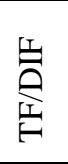 & 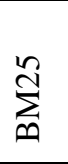 \\
\hline Baseline & 2.11 & 2.14 & 2.07 & 2.08 & 1.98 & 2.03 \\
\hline Custom & 2.26 & 2.30 & 2.15 & 2.21 & 2.11 & 2.07 \\
\hline All & 2.17 & 2.25 & 2.08 & 2.18 & 1.94 & 2.01 \\
\hline
\end{tabular}

Table 3. Performance Comparison: NDCG

\begin{tabular}{|c|c|c|c|c|c|c|}
\hline Evaluation & \multicolumn{2}{|c|}{ NDCG@1 } & \multicolumn{2}{|c|}{ NDCG@2 } & \multicolumn{2}{|c|}{ NDCG@3 } \\
\hline $\begin{array}{l}\text { Retrieval } \\
\text { Model } \\
\text { Criteria } \\
\text { Setting } \boldsymbol{\nabla}\end{array}$ & 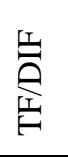 & $\sum_{n}^{n}$ & 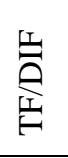 & $\sum_{\infty}^{\mathfrak{n}}$ & $\underset{⿱ 乛 龰}{\stackrel{5}{\mid c}}$ & $\sum_{\infty}^{\mathfrak{n}}$ \\
\hline Baseline & 0.81 & 8.4 & 0.79 & 8.0 & 0.78 & 0.79 \\
\hline Custom & 0.85 & 8.9 & 0.82 & 8.3 & 0.83 & 0.80 \\
\hline All & 0.83 & 8.7 & 0.80 & 8.3 & 0.75 & 0.76 \\
\hline
\end{tabular}

\section{Conclusion}

In this paper, we proposed a hybrid method for Ph.D. supervisor selection, which uses detailed domain specific knowledge, keeping the student's needs in mind. The proposed method retrieves potential supervisors based on the custom selection of criteria/sub-criteria of interest of a user (i.e., a student). This differs from previous works, which retrieve potential supervisors based on all the factors of a curated list of factors, and do not give importance to the fact that not all students might be interested in all the factors. Our evaluation of the proposed method shows that allowing users to select the criteria/sub-criteria of interest provides more satisfaction in the recommendations than recommendations generated purely based on research area relevance and recommendations generated considering all criteria/sub-criteria. Evaluations also shows that the Okapi BM25 based recommendations perform better than the TF/IDF based recommendation. Several of the previous methods are not easily scalable, as they require that students perform pairwise comparisons of the professors with respect to the considered criteria/sub-criteria [1] [4] [5]. Our method is easily scalable to larger datasets, however, as it evaluates and ranks the professors automatically based on the defined metrics.

\section{References}

[1] Momeni, M., Samimi, B., Afshari, M. A., Maleki, M. H., \& Mohammadi, J. (2011). Selection process of supervisor for doctoral dissertation using analytical network process (ANP): An Iranian study. Journal of Management \& Strategy,2(2), 63. [2] Manderson, D. (1996). Asking better questions: approaching the process of thesis supervision. Journal of Legal Education, 46(3), 407-419.

[3] Phillips, E., \& Pugh, D. (2010). How to get a PhD: A handbook for students and their supervisors. McGraw-Hill Education (UK).

[4] Ray, S., \& Marakas, G. (2007). Selecting a doctoral dissertation supervisor: Analytical hierarchy approach to the multiple criteria problem. International Journal of Doctoral Studies, 2(1), 23-32.

[5] Datta, S., Beriha, G. S., Patnaik, B., \& Mahapatra, S. S. (2009). Use of compromise ranking method for supervisor selection: A MCDM approach. International Journal of Vocational and Technical Education, 1(1), 007-013.

[6] Zhang, M., Ma, J., Liu, Z., Sun, J., \& Silva, T. (2016). A research analytics framework-supported recommendation approach for supervisor selection. British Journal of Educational Technology, 47(2), 403-420.

[7] Zhang, M., Sun, J., Ma, J., Wu, T., \& Liu, Z. (2016, January). A Personality Matching-aided Approach for Supervisor Recommendation (research-in-progress). In 2016 49th Hawaii International Conference on System Sciences (HICSS) (pp. 678-687). IEEE.

[8] Beel, J., Gipp, B., Langer, S., \& Breitinger, C. (2016). paper recommender systems: a literature survey. International Journal on Digital Libraries, 17(4), 305-338.

[9] Sugiyama, K., \& Kan, M. Y. (2010, June). Scholarly paper recommendation via user's recent research interests. In Proceedings of the 10th annual joint conference on Digital libraries (pp. 29-38). ACM.

[10] Ferrara, F., Pudota, N., \& Tasso, C. (2011, January). A keyphrase-based paper recommender system. In Italian Research Conference on Digital Libraries (pp. 14-25). Springer, Berlin, Heidelberg.

[11] Hwang, S. Y., Hsiung, W. C., \& Yang, W. S. (2003). A prototype WWW literature recommendation system for digital libraries. Online Information Review, 27(3), 169-182.

[12] Pennock, D. M., Horvitz, E., Lawrence, S., \& Giles, C. L. (2000, June). Collaborative filtering by personality diagnosis: A hybrid memory-and model-based approach. In Proceedings of the 16th conference on Uncertainty in artificial intelligence (pp. 473-480). Morgan Kaufmann Publishers Inc. 
[13] Chen, H. H., Gou, L., Zhang, X., \& Giles, C. L. (2011, June). Collabseer: a search engine for collaboration discovery. In Proceedings of the 11th annual international ACM/IEEE joint conference on Digital libraries (pp. 231-240). ACM.

[14] Gollapalli, S. D., Mitra, P., \& Giles, C. L. (2012, June). Similar researcher search in academic environments. In Proceedings of the 12th ACM/IEEE-CS joint conference on Digital Libraries (pp. 167-170). ACM.

[15] Kong, X., Jiang, H., Yang, Z., Xu, Z., Xia, F., \& Tolba, A. (2016). Exploiting publication contents and collaboration networks for collaborator recommendation. PloS one, 11(2).

[16] Chaiwanarom, P., \& Lursinsap, C. (2015). Collaborator recommendation in interdisciplinary computer science using degrees of collaborative forces, temporal evolution of research interest, and comparative seniority status. Knowledge-Based Systems, 75, 161-172.

[17] Han, S., He, D., Jiang, J., \& Yue, Z. (2013, October). Supporting exploratory people search: a study of factor transparency and user control. In Proceedings of the 22nd ACM international conference on Information \& Knowledge Management (pp. 449-458). ACM.

[18] McDougall, D., \& Macdonald, C. (2009, July). Expertise search in academia using facets. In Proceedings of the 32nd international ACM SIGIR conference on Research and development in information retrieval (pp. 834-834). ACM.

[19] Alarfaj, F., Kruschwitz, U., Hunter, D., \& Fox, C. (2012, June). Finding the right supervisor: expert-finding in a university domain. In Proceedings of the 2012 Conference of the North American Chapter of the Association for Computational Linguistics(ACL): human language technologies: student research workshop (pp. 1-6). ACL

[20] Gormley, C., \& Tong, Z. (2015). Elasticsearch: The Definitive Guide: A Distributed Real-Time Search and Analytics Engine. " O'Reilly Media, Inc.".

[21] Saaty, T. (1980). The analytic process: planning, priority setting, resources allocation. New York: McGraw.

[22] Buckley, J. J. (1985). Fuzzy hierarchical analysis. Fuzzy sets and systems, 17(3), 233-247.

[23] Ayhan, M. B. (2013). A fuzzy AHP approach for supplier selection problem: A case study in a Gear motor company. arXiv preprint arXiv:1311.2886.

[24] Lafleur, J. M. (2011, March). Probabilistic AHP and TOPSIS for multi-attribute decision-making under uncertainty. In Aerospace Conference, 2011 IEEE (pp. 1-18). [25] Mimović, P., Stanković, J., \& Janković Milić, V. (2015). Decision-making under uncertainty-the integrated approach of the AHP and Bayesian analysis. Economic researchEkonomska istraživanja, 28(1), 868-878.

[26] Bryson, N., \& Mobolurin, A. (1997). An action learning evaluation procedure for MCDM problems. European Journal of Operational Research, 96(2), 379-386.

[27] Bryson, N., \& Joseph, A. (2000). Generating consensus priority interval vectors for group decision-making in the AHP. Journal of Multi-Criteria Decision Analysis, 9(4), 127 137.

[28] Lin, H. F. (2010). An application of fuzzy AHP for evaluating course website quality. Computers \& Education, 54(4), 877-888.

[29] Klir, G. J., \& Yuan, B. (1996). Fuzzy sets, fuzzy logic, and fuzzy systems: selected papers by Lotfi A. Zadeh. World Scientific Publishing Co., Inc.
[30] Kaufman, A., \& Gupta, M. M. (1991). Introduction to fuzzy arithmetic. New York, USA: Van Nostrand Reinhold Company.

[31] Lops, P., De Gemmis, M., \& Semeraro, G. (2011). Content-based recommender systems: State of the art and trends. In Recommender systems handbook (pp. 73-105). Springer, Boston, MA.

[32] Henrich, A., \& Wegmann, M. (2017). Searching an Appropriate Journal for your Paper-an Approach Inspired by Expert Search and Data Fusion.

[33] Robertson, S., \& Zaragoza, H. (2009). The probabilistic relevance framework: BM25 and beyond. Foundations and Trends ${ }^{\circledR}$ in Information Retrieval, 3(4), 333-389.

[34] Chudnovsky, D., López, A., Rossi, M. A., \& Ubfal, D. (2008). Money for science? The impact of research grants on academic output. Fiscal Studies, 29(1), 75-87.

[35] Jauch, L. R. (1988). Evaluation of University Professors' Research Performance: Management Science, 3(4), 476.

[36] Ethington, C. A., \& Pisani, A. (1993). The RA and TA experience: Impediments and benefits to graduate study. Research in Higher Education, 34(3), 343-354.

[37] Wong, H. Y., \& Sanders, J. M. (1983). Gender differences in the attainment of doctorates. Sociological Perspectives, 26(1), 29-50.

[38] Bozeman, B., \& Gaughan, M. (2007). Impacts of grants and contracts on academic researchers' interactions with industry. Research policy, 36(5), 694-707.

[39] Lee, S., \& Bozeman, B. (2005). The impact of research collaboration on scientific productivity. Social studies of science, 35(5), 673-702.

[40] de Solla Price, D. J., \& Beaver, D. (1966). Collaboration in an invisible college. American psychologist, 21(11), 1011. [41] Rokach, L., Mitra, P., Kataria, S., Huang, W., \& Giles, L. (1978). A supervised learning method for context-aware citation recommendation in a large corpus. INVITED SPEAKER: Analyzing the Performance of Top-K Retrieval Algorithms, 1978.

[42] Sinha, A., Shen, Z., Song, Y., Ma, H., Eide, D., Hsu, B. J. P., \& Wang, K. (2015, May). An overview of microsoft academic service (mas) and applications. In Proceedings of the 24th international conf. on $W W W$ (pp. 243-246). ACM.

[43] Beel, J., Langer, S., Genzmehr, M., \& Nürnberger, A. (2013, July). Introducing Docear's research paper recommender system. In Proceedings of the 13th ACM/IEEECS joint conference on Digital libraries (pp. 459-460). ACM. [44] Uchiyama, K., Nanba, H., Aizawa, A., \& Sagara, T. (2011). OSUSUME: cross-lingual recommender system for research papers. In Proc. of the 2011 Workshop on Contextawareness in Retrieval \& Recommendation (39-42). ACM.

[45] Ives, G., \& Rowley, G. (2005). Supervisor selection or allocation and continuity of supervision: Ph. D. students' progress and outcomes. Studies in Higher Education, 30(5), 535-555.

[46] Yang, K. W., \& Huh, S. Y. (2008). Automatic expert identification using a text categorization technique in knowledge management systems. Expert Systems with Applications, 34(2), 1445-1455.

[47] Hasan, M. A., \& Schwartz, D. G. (2018). RecAdvisor: Criteria-based $\mathrm{Ph}$. D. Supervisor Recommendation. In The 41st International ACM SIGIR Conference on Research \& Development in Information Retrieval (pp. 1325-1328). ACM. 